\title{
Construction of a high-density genetic linkage map and QTL mapping of oleic acid content and three agronomic traits in sunflower (Helianthus annuus L.) using specific-locus amplified fragment sequencing (SLAF-seq)
}

\author{
Fei Zhou ${ }^{1,2)}$, Yan Liu ${ }^{1,2)}$, Chunbo Liang ${ }^{2)}$, Wenjun Wang ${ }^{2)}$, Cen $\mathrm{Li}^{2)}$, Yongli Guo ${ }^{2)}$, Jun $\mathrm{Ma}^{2)}$, Ying $\mathrm{Yu}^{2)}$, \\ Lijuan Fan $^{2)}$, Yubo Yao ${ }^{2)}$, Dongsheng Zhao ${ }^{2)}$, Xuemei Liu ${ }^{1)}$ and Xutang Huang*2) \\ 1) College of Life Science, Northeast Forestry University, Harbin, 150040, China \\ 2) Institute of Industrial Crops, Heilongjiang Academy of Agricultural Sciences, Harbin, 150086, China
}

\begin{abstract}
High-density genetic linkage maps are particularly important for quantitative trait loci (QTL) mapping, genome assembly, and marker-assisted selection (MAS) in plants. In this study, a high-density genetic linkage map of sunflower (Helianthus annuus L.) was constructed using an $\mathrm{F}_{2}$ population generated from a cross between Helianthus annuus L. '86-1' and 'L-1-OL-1' via specific-locus amplified fragment sequencing (SLAFseq). After sequence preprocessing, $530.50 \mathrm{M}$ reads $(105.60 \mathrm{~Gb})$ were obtained that contained a total of 343,197 SLAFs, of which 39,589 were polymorphic. Of the polymorphic SLAFs, 6,136 were organized into a linkage map consisting of 17 linkage groups (LGs) spanning 2,221.86 cM, with an average genetic distance of $0.36 \mathrm{cM}$ between SLAFs. Based on this high-density genetic map, QTL analysis was performed that focused on four sunflower phenotypic traits: oleic acid content (OAC), plant height (PH), head diameter (HD), and stem diameter (SD). Subsequently, for these four traits eight QTLs were detected that will likely be useful for increasing our understanding of genetic factors underlying these traits and for use in marker-assisted selection (MAS) for future sunflower breeding.
\end{abstract}

Key Words: Helianthus annuus L., high-density genetic map, SLAF-seq, SNP, oleic acid content, agronomic traits, QTL.

\section{Introduction}

Sunflower (Helianthus annuus L.) is one of the most important vegetable oil sources worldwide. Cultivated sunflower is a diploid species $(2 \mathrm{n}=2 \mathrm{x}=34)$ with a large genome size of $3.6 \mathrm{~Gb}$ (Badouin et al. 2017). Between 2007 and 2016, approximately 24.800 million hectares of sunflower crop were cultivated globally per annum, of which approximately 0.932 million hectares were cultivated in China (Food and Agricultural Organization of the United Nations Statistical Databases 2017; http://www.fao.org/faostat/en/\#home).

Genetic maps are a key resource for understanding genome organization and evolutionary relationships (Wang et al. 2011) and are particularly valuable tools for QTL mapping and marker-assisted selection (MAS). Because many important agronomic traits of sunflower are controlled by numerous loci, quantitative trait locus (QTL) analysis and

Communicated by Akemi Ohmiya

Received April 17, 2018. Accepted September 18, 2018.

First Published Online in J-STAGE on November 21, 2018.

*Corresponding author (e-mail: hxthlj@126.com)
MAS can shorten breeding duration and accelerate breeding efficacy of new sunflower varieties. To date, several sunflower genetic linkage maps incorporating different molecular markers have been constructed based on restriction fragment length polymorphisms (RFLPs) (Berry et al. 1995, Gentzbittel et al. 1995, Jan et al. 1998), random amplification of polymorphic DNA (RAPD) (Rieseberg et al. 1993), target region amplification polymorphisms (TRAPs) (Bing et al. 2008, Maleki et al. 2014), amplified fragment length polymorphisms (AFLPs) (Kusterer et al. 2004, Maleki et al. 2014), simple sequence repeats (SSRs) (Talia et al. 2010, Tang et al. 2002, Yu et al. 2003), single nucleotide polymorphisms (SNPs) (Hulke et al. 2015), and sequence-related amplification polymorphisms (SRAPs) (Lyu et al. 2017). Until recently, most such maps were based on low-throughput marker technologies, which resulted in genetic maps of limited density. These older maps have since been eclipsed by higher density maps generated using new high-throughput genotyping technologies such as restriction site-associated DNA (RAD) tag sequencing (Talukder et al. 2014). Nevertheless, with the recent release of the sunflower genome (Badouin et al. 2017) and the development of genome 
sequencing technologies, soon SNPs will be the predominant markers used for construction of genetic maps and for marker-assisted breeding. Indeed, as single nucleotide DNA sequence variants, SNPs are currently markers of choice for high-density genetic map construction due to their abundance, uniform genome distribution, and low-cost detection (Ganal et al. 2009).

Plant height, head diameter, and stem diameter are important agronomic traits in sunflower, with known effects on yield and lodging, while fatty acid composition influences the quality and stability of sunflower oil. One advantage of sunflower oil, which is high in oleic acid, is its high degree of oxidative stability that is desirable for cooking, refining, and storage uses, as compared to oils low in oleic acid (Fuller et al. 1967). To date, most reported molecular markers related to sunflower plant height, head diameter, stem diameter, and oleic acid content have been nonfunctional markers and even false positive QTLs, impeding progress in marker-assisted selection (MAS). The construction of a high-density SNP map of sunflower will improve the efficiency and accuracy of localization of important sunflower QTLs to benefit future in-depth discovery and development of functional markers and MAS in sunflower.

SLAF-seq is a new technology that can be used to rapidly develop SNP markers after construction of a SLAF-seq library (Sun et al. 2013). Due to its advantages of highthroughput, high accuracy, low cost, and short cycle times, SLAF-seq technology has been successfully applied to construct high-density maps of watermelon (Shang et al. 2016), flue-cured tobacco (Gong et al. 2016), pear (Wang et al. 2017b), soybean (Li et al. 2017), cucumber (Xu et al. 2015), rice (Peng et al. 2016), sorghum (Ji et al. 2017), and grape (Wang et al. 2017a).

In this study, to achieve rapid mass discovery of SNP markers and high-density genetic mapping of sunflower, two elite sunflower (Helianthus annuus L.) cultivars '86-1' and 'L-1-OL-1' were used as female and male parents, respectively. Next, SLAF-seq technology was used to develop SLAF markers using genetic material from both the $\mathrm{F}_{2}$ hybrid population and parents to construct a high-density linkage map. Based on this high-density genetic map, QTLs associated with oleic acid content and three agronomic traits (plant height, head diameter, and stem diameter) were identified. These results will likely facilitate future development of functional markers and the application of MAS to sunflower breeding.

\section{Materials and Methods}

\section{Plant materials}

An $\mathrm{F}_{2}$ mapping population derived from a cross of sunflower inbred lines 'L-1-OL-1' (male parent, bred in Serbia) and ' $86-1$ ' (female parent, bred in China) was used to construct a linkage map. The seed of 'L-1-OL-1' and '86-1' differ significantly in oleic acid content, with 'L-1-OL-1' exhibiting higher oleic acid content (87.65\%) than ' $86-1$ '
(18.61\%). Both parents and their $84 \mathrm{~F}_{2}$ progeny were planted in the experimental field of Industrial Crops Institute, Heilongjiang Academy of Agricultural Sciences in Harbin, China.

\section{DNA extraction}

Young healthy leaves from $\mathrm{F}_{2}$ individuals and parents were sampled and genomic DNA was extracted using a DNAsecure Plant Kit (TIANGEN Biotech, Beijing, China).

\section{SLAF library construction and high-throughput sequencing}

SLAF-seq was used to genotype $84 \mathrm{~F}_{2}$ individuals and their parents, as previously described with minor modifications (Sun et al. 2013). The digestion sites and the lengths and length distribution of resulting fragments were predicted using the reference genome. Three criteria were considered: i) The number of SLAFs must be suitable for the specific needs of the research project. ii) The SLAFs must be evenly distributed throughout the sequences to be examined. iii) Repeated SLAFs must be avoided (Sun et al. 2013). The HaeIII restriction enzyme was finally chosen for use in achieving complete digestion of genomic DNA of the mapping ( $\mathrm{F}_{2}$ population) (New England Biolabs, USA) followed by addition of a single-nucleotide (A) overhang to each digested fragment using Klenow Fragment $\left(3^{\prime} \rightarrow 5^{\prime}\right.$ exo $\left.^{-}\right)$ (NEB) and dATP at $37^{\circ} \mathrm{C}$. Duplex tag-labeled sequencing adapters (PAGE-purified, Life Technologies, USA) were then ligated to the A-tailed fragments using T4 DNA ligase. Polymerase chain reaction (PCR) was performed using diluted restriction enzyme-digested and ligated DNA samples, dNTP, Q5 ${ }^{\circledR}$ High-Fidelity DNA Polymerase (NEB), and PCR primers (Forward primer: 5'-AATGATACGGCGAC CACCGA-3', reverse primer: 5'-CAAGCAGAAGACGG CATACG-3') (PAGE purified, Life Technologies). PCR products were then purified using Agencourt AMPure XP beads (Beckman Coulter, High Wycombe, UK) and pooled. Pooled samples were separated by $2 \%$ agarose gel electrophoresis. Fragments ranging from 314 to $444 \mathrm{bp}$ in size (including indexes and adaptors) were excised and purified using a QIAquick Gel Extraction Kit (Qiagen, Hilden, Germany). Gel-purified products were then quantified and appropriately diluted. Paired-end sequencing (of each end of $125 \mathrm{bp}$ ) was performed using an Illumina HiSeq 2500 system (Illumina, Inc., San Diego, CA, USA) according to the manufacturer's recommendations.

\section{Sequence data grouping and genotyping}

SLAF marker identification and genotyping were performed using procedures previously described (Sun et al. 2013). The genome of Helianthus annuus L. (Badouin et al. 2017) (https://www.ncbi.nlm.nih.gov/genome/?term= Helianthus+annuus\%5Borgn\%5D) was used as a reference. Briefly, low-quality reads (quality score $<30$ ) were filtered out and remaining raw reads were assigned to each progeny according to duplex barcode sequences. After the barcodes and terminal 5-bp sequences were trimmed from each 
high-quality read, clean reads were clustered together according to their sequence identities. Sequences mapping to the same locus with over $90 \%$ identity were defined as one SLAF locus (Zhang et al. 2015). Single nucleotide polymorphic (SNP) loci for each SLAF locus were then assigned to each parent and SLAFs with more than 3 SNPs between parents were filtered out first. Alleles of each SLAF locus were then defined from parental reads with sequence depth $>10$-fold. For diploid species, one SLAF locus can contain a maximum of four genotypes; therefore, SLAF loci with more than four alleles were defined as repetitive SLAFs and were discarded. Only SLAFs with two to four alleles were defined here as polymorphic and considered as potential markers.

All polymorphic SLAFs loci were genotyped based on their consistency within both parental and offspring SNP loci. Marker codes of polymorphic SLAFs were next analyzed according to the population type $\mathrm{F}_{2}$, which consisted of one segregation type $(a \mathrm{a} \times \mathrm{bb})$. Genotype scoring was then performed using a Bayesian approach to further ensure genotype quality (Sun et al. 2013). Subsequently, highquality SLAF markers for genetic mapping were retained using the following criteria. First, average sequence depths should be $>10$-fold in the parents. Second, markers with more than 30\% missing data were filtered out and discarded. Third, the chi-square test was performed to examine the severity of segregation distortion. Markers with significant segregation distortion $(p<0.01)$ were excluded from map construction.

\section{High-density genetic map construction}

Marker loci were partitioned primarily into linkage groups (LGs) using modified logarithm of odds (MLOD) score values of $>5$ as a cutoff value. To ensure efficient construction of the high-density and high-quality map, the HighMap strategy was utilized to arrange SLAF markers in a specific order and correct genotyping errors within LGs (Liu et al. 2014). Specifically, the genetic map was constructed according to the maximum likelihood method (Van Ooijen 2011) and genotyping errors were corrected using the SMOOTH algorithm (Van Os et al. 2005). A k-nearest neighbor algorithm was applied to impute missing genotypes (Huang et al. 2011). Finally, genetic map distances were estimated using the Kosambi mapping function (Kosambi 1943).

\section{Phenotypic data collection}

The oleic acid content values of seeds were measured at the Oil Crops Research Institute, China Academy of Agricultural Sciences using gas chromatography (model Agilent 7890A, USA). Plant height (PH), head diameter (HD), and stem diameter (SD) were measured during the full flowering period before harvest. The stem diameter was measured through the center of each stem.

\section{QTL analysis using a high-density genetic map}

QTLs that mapped to oleic acid content and the three other aforementioned agronomic traits were identified using the composite interval mapping (CIM) method application within R/qtl software. The threshold of LOD scores for evaluating the statistical significance of QTL effects was determined using 1,000 permutations. Based on these permutations, a LOD score of 2.0 was used as the minimum cutoff for declaring the presence of a QTL within a particular genomic region.

\section{Availability of data}

The dataset supporting the conclusions of this article has been available in The European Nucleotide Archive as accession number PRJEB25920 (https://www.ebi.ac.uk/ena/ submit/sra/).

\section{Results}

Analysis of SLAF sequencing data and SLAF markers

A total of $105.60 \mathrm{~Gb}$ of raw reads consisting of $530.50 \mathrm{Mb}$ paired-end reads were obtained using highthroughput sequencing of SLAF libraries. Among those reads, the average percentage of Q30 bases (bases with a quality score of 30 , indicating a $1 \%$ chance of an error and thus a $99 \%$ confidence level) was $90.84 \%$ and the GC content was $44.36 \%$ (Supplemental Table 1). There were $4,505,523$ reads from the female parent and 4,839,674 reads from the male parent and an average number of reads for the $\mathrm{F}_{2}$ population of $2,488,845$. SLAF numbers obtained from the male and female parents were 266,226 and 252,824 , respectively (Table 1). The average sequencing depth was 18.18-fold for the male parent and 17.82-fold for the female parent. In the $F_{2}$ population, the average number of SLAF markers was 258,665 and the average coverage was 9.62fold (Fig. 1). After read clustering was completed, a total of 343,197 SLAFs were detected.

Among the 343,197 SLAFs obtained, 39,589 were polymorphic, with a polymorphism rate of $11.54 \%$, while the remaining 303,045 were non-polymorphic and 563 were repetitive. After filtering out SLAF markers lacking parental information, 27,261 polymorphic markers were successfully classified into eight segregation patterns (Fig. 2). The $\mathrm{F}_{2}$ population was obtained by selfing of the $F_{1}$ progeny obtained from a cross between two parents with homozygous genotype of aa or bb. Consequently, only $\mathrm{F}_{2}$ plants with the aa $\times$ bb segregation pattern were used for further analysis, yielding a total of 19,282 markers that fell within this segregation pattern type. Among these 19,282 markers, 6,136

Table 1. Summary of marker depths

\begin{tabular}{lccc}
\hline \hline Samples & Marker numbers & Total depth & Average depth \\
\hline L-1-OL-1 (P) & 266,226 & $4,839,674$ & 18.18 \\
86-1 (M) & 252,824 & $4,505,523$ & 17.82 \\
Offspring & 258,665 & $2,488,845$ & 9.62 \\
\hline
\end{tabular}




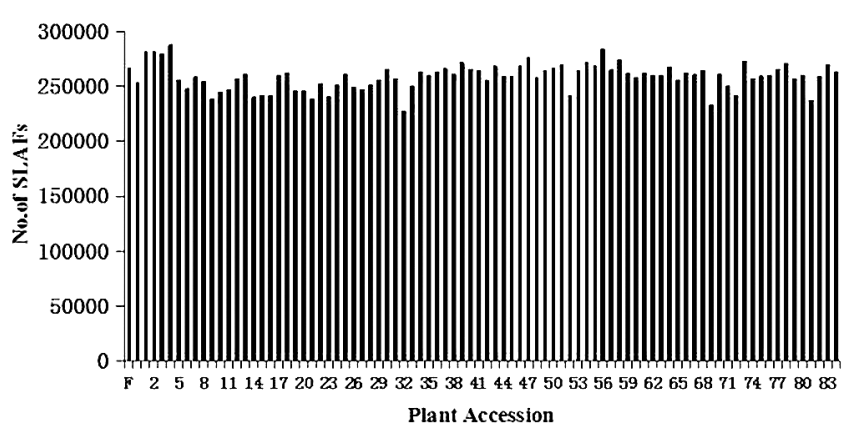

(B)

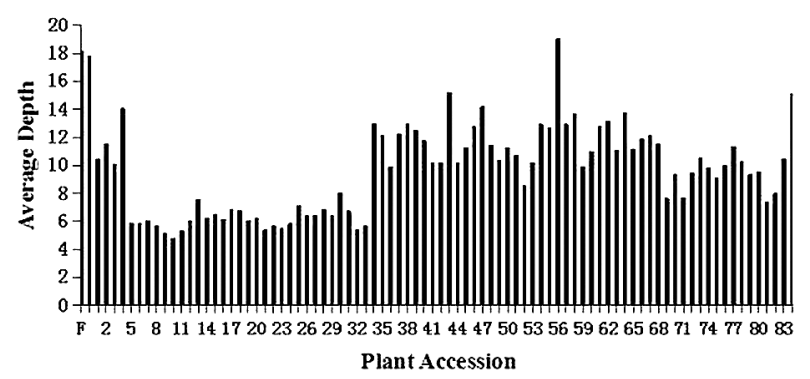

Fig. 1. Number of SLAF markers (A) and average sequencing depth (B) for each $\mathrm{F}_{2}$ individual and parents. The $\mathrm{x}$-axis indicates the plant accession including female parent $(\mathrm{F})$, male parent $(\mathrm{M})$, and each of the $F_{2}$ individuals; the $y$-axis indicates the number of SLAF markers (A) and the average sequencing coverage (B).

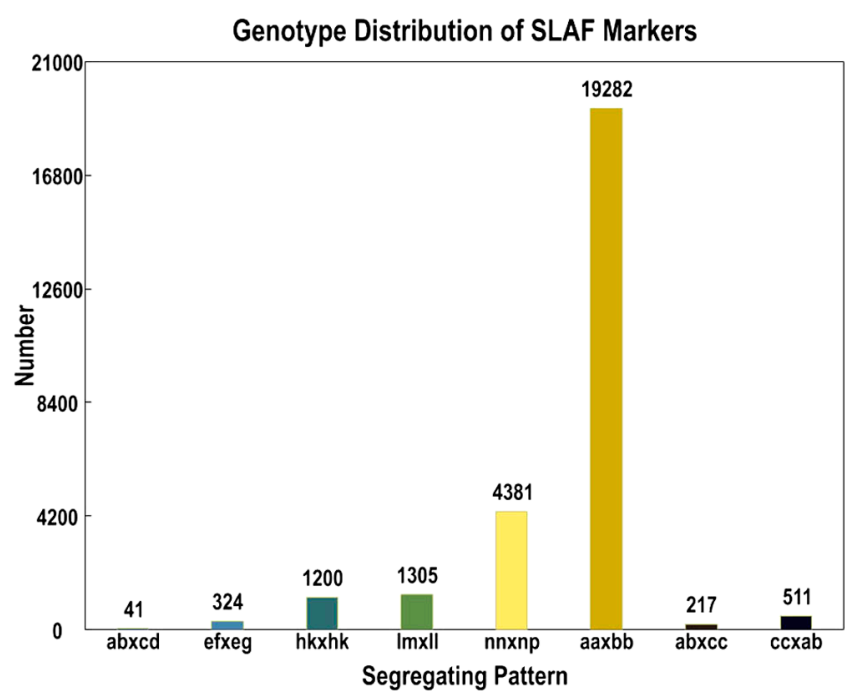

Fig. 2. Number of polymorphic SLAF markers in each of eight segregation patterns. The $\mathrm{x}$-axis indicates eight segregation patterns of polymorphic SLAF markers; the $y$-axis indicates the number of markers.

markers were used for construction of the final genetic map after discarding the remainder, which were homozygous between parents. Ultimately, a sequence depth for the parents of greater than 10-fold was achieved, with over $70 \%$ integrity of SLAF tags and exclusion from the final map of

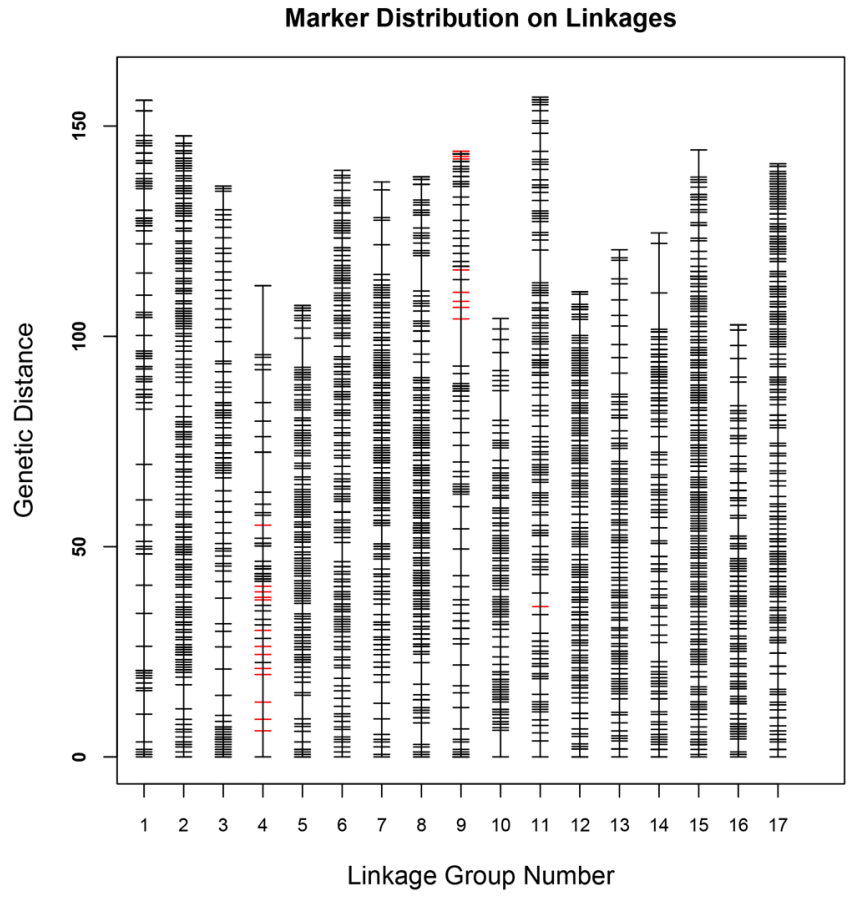

Fig. 3. Distribution of SLAF markers on the 17 linkage groups of sunflower. A black bar indicates a SLAF marker. A red bar indicates a segregation distortion marker. The $\mathrm{x}$-axis represents the linkage group number and the $y$-axis indicates the genetic distance $(\mathrm{cM})$ in each linkage group.

markers showing extremely significant $(\mathrm{p}<0.01)$ segregation distortion.

\section{Basic characteristics of the genetic map}

In total, 6,136 markers were grouped into 17 linkage groups (LGs). The average integrity of mapped markers was $99.79 \%$, indicating an acceptably high genetic map quality (Supplemental Tables 2-4). The total genetic length of the map was $2221.86 \mathrm{cM}$, with an average distance between adjacent markers of $0.36 \mathrm{cM}$ (Fig. 3). All LGs are shown in Table 2. On average, each LG contained 361 markers that spanned an average of $130.70 \mathrm{cM}$. The lengths of LGs ranged from $102.72 \mathrm{cM}$ (LG16) to $156.86 \mathrm{cM}$ (LG11). LG11, the longest LG, had a genetic length of $156.86 \mathrm{cM}$ and contained 239 markers with an average distance between markers of $0.66 \mathrm{cM}$. LG16, the shortest LG, had a genetic length $102.72 \mathrm{cM}$ and contained 597 markers, with an average inter-marker distance of $0.17 \mathrm{cM}$. LG17 contained the maximum number of markers (801), whereas LG4 possessed the minimum marker number (78). The "Gap $\leq 5$ " value ranged from $92.49 \%$ to $100 \%$, with a total of 44 gaps of length $>5 \mathrm{cM}$. Of these, a total of 40 gaps were 5 to $10 \mathrm{cM}$ in length and four gaps had lengths $>10 \mathrm{cM}$. The largest gap on this map, $16.40 \mathrm{cM}$ in length, was located within LG4. Most gaps (13) of lengths greater than $5 \mathrm{cM}$ were present within LG1, while LG6, LG12, and LG17 contained no gap with length greater than $5 \mathrm{cM}$ (Table 2, Supplemental Table 4). A total of 11,980 SNP 
Table 2. Description on basic characteristics of the seventeen linkage groups

\begin{tabular}{|c|c|c|c|c|c|c|}
\hline Linkage group & $\begin{array}{c}\text { Total number of } \\
\text { marker }\end{array}$ & $\begin{array}{c}\text { Total distance } \\
(\mathrm{cM})\end{array}$ & $\begin{array}{c}\text { Average distance } \\
(\mathrm{cM})\end{array}$ & Gap $\leq 5 \mathrm{cM}$ & Max gap (cM) & $\begin{array}{c}\text { Number of markers with } \\
\text { segregation distortion }\end{array}$ \\
\hline LG1 & 174 & 156.11 & 0.90 & $92.49 \%$ & 13.15 & 0 \\
\hline LG2 & 354 & 147.69 & 0.42 & $99.72 \%$ & 5.74 & 0 \\
\hline LG3 & 128 & 135.70 & 1.07 & $97.64 \%$ & 6.25 & 0 \\
\hline LG4 & 78 & 112.03 & 1.45 & $93.51 \%$ & 16.40 & 14 \\
\hline LG5 & 321 & 107.33 & 0.34 & $99.38 \%$ & 6.95 & 0 \\
\hline LG6 & 519 & 139.47 & 0.27 & $100.00 \%$ & 4.54 & 0 \\
\hline LG7 & 370 & 136.72 & 0.37 & $98.92 \%$ & 7.07 & 0 \\
\hline LG8 & 746 & 137.19 & 0.19 & $99.60 \%$ & 8.41 & 0 \\
\hline LG9 & 239 & 143.97 & 0.60 & $97.90 \%$ & 11.20 & 10 \\
\hline LG10 & 178 & 104.25 & 0.59 & $98.87 \%$ & 7.07 & 0 \\
\hline LG11 & 239 & 156.86 & 0.66 & $99.58 \%$ & 7.80 & 1 \\
\hline LG12 & 387 & 110.59 & 0.29 & $100.00 \%$ & 3.82 & 0 \\
\hline LG13 & 186 & 120.58 & 0.65 & $99.46 \%$ & 5.02 & 0 \\
\hline LG14 & 194 & 124.59 & 0.65 & $98.96 \%$ & 11.76 & 0 \\
\hline LG15 & 625 & 144.32 & 0.23 & $99.84 \%$ & 6.46 & 0 \\
\hline LG16 & 597 & 102.72 & 0.17 & $99.83 \%$ & 5.62 & 0 \\
\hline LG17 & 801 & 141.02 & 0.18 & $100.00 \%$ & 3.70 & 0 \\
\hline Total & 6,136 & 2221.86 & 0.36 & $98.57 \%$ & 16.40 & 0 \\
\hline
\end{tabular}

"Gap $\leq 5$ " indicated the percentages of gaps in which the distance between adjacent markers was smaller than or equal to $5 \mathrm{cM}$.

Table 3. Distribution of SNP loci on the 17 linkage groups of sunflower

\begin{tabular}{lcrrc}
\hline \hline $\begin{array}{l}\text { Linkage } \\
\text { group ID }\end{array}$ & SNP number & Tri & Trv & Tri/Trv \\
\hline LG1 & 357 & 231 & 126 & 1.83 \\
LG2 & 742 & 496 & 246 & 2.02 \\
LG3 & 269 & 188 & 81 & 2.32 \\
LG4 & 131 & 81 & 50 & 1.62 \\
LG5 & 593 & 401 & 192 & 2.09 \\
LG6 & 1090 & 710 & 380 & 1.87 \\
LG7 & 631 & 425 & 206 & 2.06 \\
LG8 & 1455 & 991 & 464 & 2.14 \\
LG9 & 447 & 299 & 148 & 2.02 \\
LG10 & 402 & 271 & 131 & 2.07 \\
LG11 & 478 & 317 & 161 & 1.97 \\
LG12 & 725 & 486 & 239 & 2.03 \\
LG13 & 428 & 277 & 151 & 1.83 \\
LG14 & 453 & 304 & 149 & 2.04 \\
LG15 & 1162 & 767 & 395 & 1.94 \\
LG16 & 1116 & 761 & 355 & 2.14 \\
LG17 & 1501 & 1016 & 485 & 2.09 \\
\hline
\end{tabular}

loci were identified in this genetic map (Table 3). LG17 contained the maximum number of SNP loci (1501), while LG4 contained the minimum number of SNP loci (131).

\section{Quality evaluation of the genetic map}

The quality of the sunflower genetic map was evaluated using both haplotype and heat mapping. Haplotype maps were developed for the parental controls and their 84 offspring using 6,136 SLAF markers, which reflected double crossover and deletion events for the $F_{2}$ population (Supplemental Data 1), with most recombination blocks distinctly defined. The missing data for each $L G$ ranged from $0.17 \%$ (LG16) to $1.45 \%$ (LG4) and did not ultimately and significantly affect the quality of the genetic map. Most of the LGs were uniformly distributed, suggesting these genet- ic maps were of high quality. Heat maps were generated using pair-wise recombination values for the 6,136 mapped SLAFs (Supplemental Data 2), since heat maps may reflect the recombination relationship between markers from each LG and are useful for finding potential ordering errors. Notably, heat maps developed here indicate that the ordering of SLAF markers within most LGs was correct.

\section{Segregation distortion of markers on the map}

Only $25(0.41 \%)$ of the 6,136 markers on the genetic map exhibited significant segregation distortion $(p<0.01)$. Notably, markers exhibiting segregation distortion were only distributed within three LGs, LG4 (17.95\%), LG9 (4.18\%), and LG11 (0.42\%). No significant correlation was found between the distribution of mapped markers and distorted markers. For example, LG17 contained the maximum number of markers (801 markers) and covered $141.02 \mathrm{cM}$, but did not contain any distorted markers. For comparison, LG4, which possessed the minimum SLAF marker number (78 markers) and covered $112.03 \mathrm{cM}$, contained 14 distorted markers.

\section{Phenotypic analysis of oleic acid content and 3 agronomic traits}

Phenotypic data for the two parents and $\mathrm{F}_{2}$ families are shown in Table 4. Of these, plant height shows the minimum coefficient of variance (CV) of $9.78 \%$, while oleic acid content exhibits the maximum CV of $39.43 \%$. The frequency distributions of four phenotypic traits were also analyzed and almost all exhibited continuous and normal distributions, with the exception of oleic acid content (Fig. 4, Table 4).

\section{QTL analysis using the high-density genetic map}

Based on the high-density genetic map, QTLs for plant 
Table 4. The characteristics of the phenotypic traits in sunflower $F_{2}$ mapping population

\begin{tabular}{|c|c|c|c|c|c|c|c|c|c|}
\hline \multirow[b]{2}{*}{ Traits } & \multicolumn{5}{|c|}{ Parents } & \multicolumn{4}{|c|}{$\mathrm{F}_{2}$ population } \\
\hline & $\begin{array}{c}\text { L-1-OL-1 } \\
(\%)\end{array}$ & $86-1(\%)$ & $\begin{array}{c}\text { Mean } \\
\%\end{array}$ & $\underset{\%}{\operatorname{Min}}$ & $\begin{array}{c}\operatorname{Max} \\
\%\end{array}$ & $\mathrm{SD}$ & $\begin{array}{c}\text { Coeffcient of } \\
\text { variance }\end{array}$ & Kurtosis & Skewness \\
\hline Oleic acid & 87.65 & 18.61 & 57.19 & 17.32 & 89.10 & 22.55 & 0.3943 & -1.22 & -0.43 \\
\hline Plant height & 125.2 & 150.1 & 166.93 & 128.1 & 225.3 & 16.33 & 0.0978 & 1.26 & 0.29 \\
\hline Head diameter & 23.1 & 18.8 & 22.33 & 11.5 & 32.2 & 4.23 & 0.1894 & -0.10 & -0.15 \\
\hline Stem diameter & 2.19 & 2.41 & 2.49 & 1.71 & 3.63 & 0.41 & 0.1647 & 0.38 & 0.63 \\
\hline
\end{tabular}
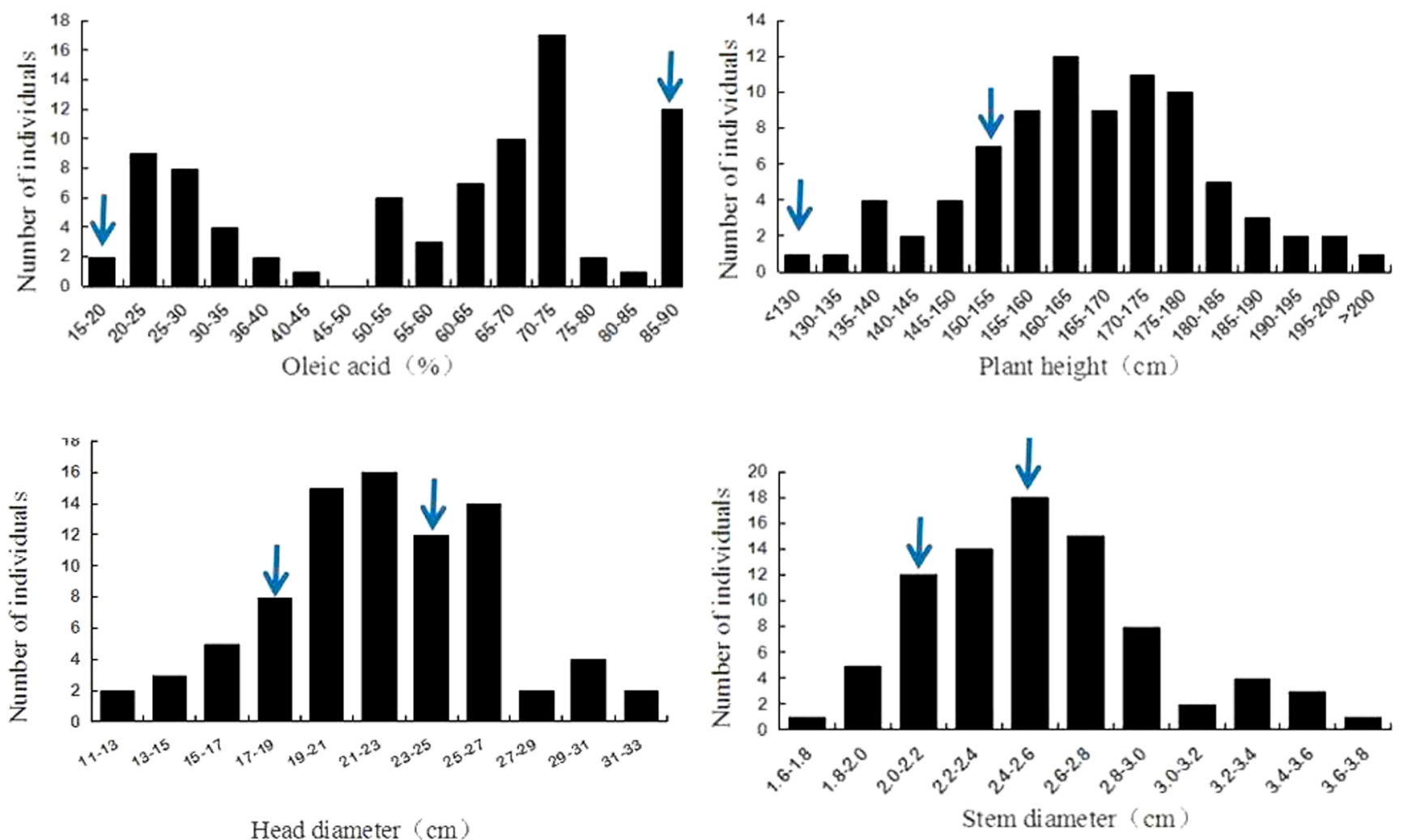

Head diameter $(\mathrm{cm})$

Fig. 4. Frequency distribution of plant height, head diameter, stem diameter and oleic acid content for the $\mathrm{F}_{2}$ families. The arrows indicate the phenotype in two parental lines (L-1-OL-1 and 86-1).

Table 5. Quantitative trait loci (QTL) analysis of sunflower the target traits

\begin{tabular}{|c|c|c|c|c|c|c|c|}
\hline Trait & QTL & LG ID & Marker number & Interval (cM) & LOD & ADD & PVE (\%) \\
\hline \multirow[t]{2}{*}{ Plant height } & PH_l & 7 & 2 & $84.223-84.223$ & 2.52 & 6.69 & 10.31 \\
\hline & PH_2 & 17 & 5 & $40.511-40.511$ & 2.50 & 7.84 & 12.28 \\
\hline \multirow[t]{2}{*}{ Head diameter } & $H D_{-}^{-} 1$ & 5 & 6 & $46.639-48.708$ & 2.78 & 1.14 & 5.63 \\
\hline & $H D_{2}^{-2}$ & 9 & 2 & $91.156-92.943$ & 2.15 & 1.28 & 5.49 \\
\hline Stem diameter & $S D \overline{1}$ & 16 & 11 & $7.266-7.266$ & 2.61 & 0.10 & 15.65 \\
\hline \multirow[t]{3}{*}{ Oleic acid } & $O \overline{A C} 1$ & 9 & 3 & $125.078-131.277$ & 2.56 & -10.81 & 12.05 \\
\hline & $O A C^{-} 2$ & 6 & 5 & $95.168-99.576$ & 2.50 & -8.41 & 5.81 \\
\hline & $\mathrm{OAC}_{-} 3$ & 6 & 3 & $109.108-109.108$ & 2.23 & -7.20 & 5.18 \\
\hline
\end{tabular}

height, head diameter, stem diameter, and oleic acid content were identified. As a result, QTLs with LOD scores above 2.0 were considered to be effective QTLs (Supplemental Fig. 1). According to the threshold, two QTLs ( $P H_{-} 1$ and $\mathrm{PH}$ 2) were detected for plant height and accounted for $10.31 \%$ and $12.28 \%$ of phenotypic variance, respectively. Two QTLs (HD_1 and HD_2) were detected for head diameter and accounted for $5.63 \%$ and $5.49 \%$ of phenotypic variance, respectively. For stem diameter, one QTL ( $S D$ 1) accounted for $15.65 \%$ of the phenotypic variance of that trait. For oleic acid, three QTLs $(O A C$ 1,OAC 2, and $O A C$ 3) accounted for $12.05 \%, 5.81 \%$, and $5.18 \%$ of phenotypic variance, respectively (Table 5). These QTLs mapped to LG5, LG6, LG7, LG9, LG16, and LG17 (Fig. 5), with LOD scores ranging from 2.15 to 2.78 (Table 5). 

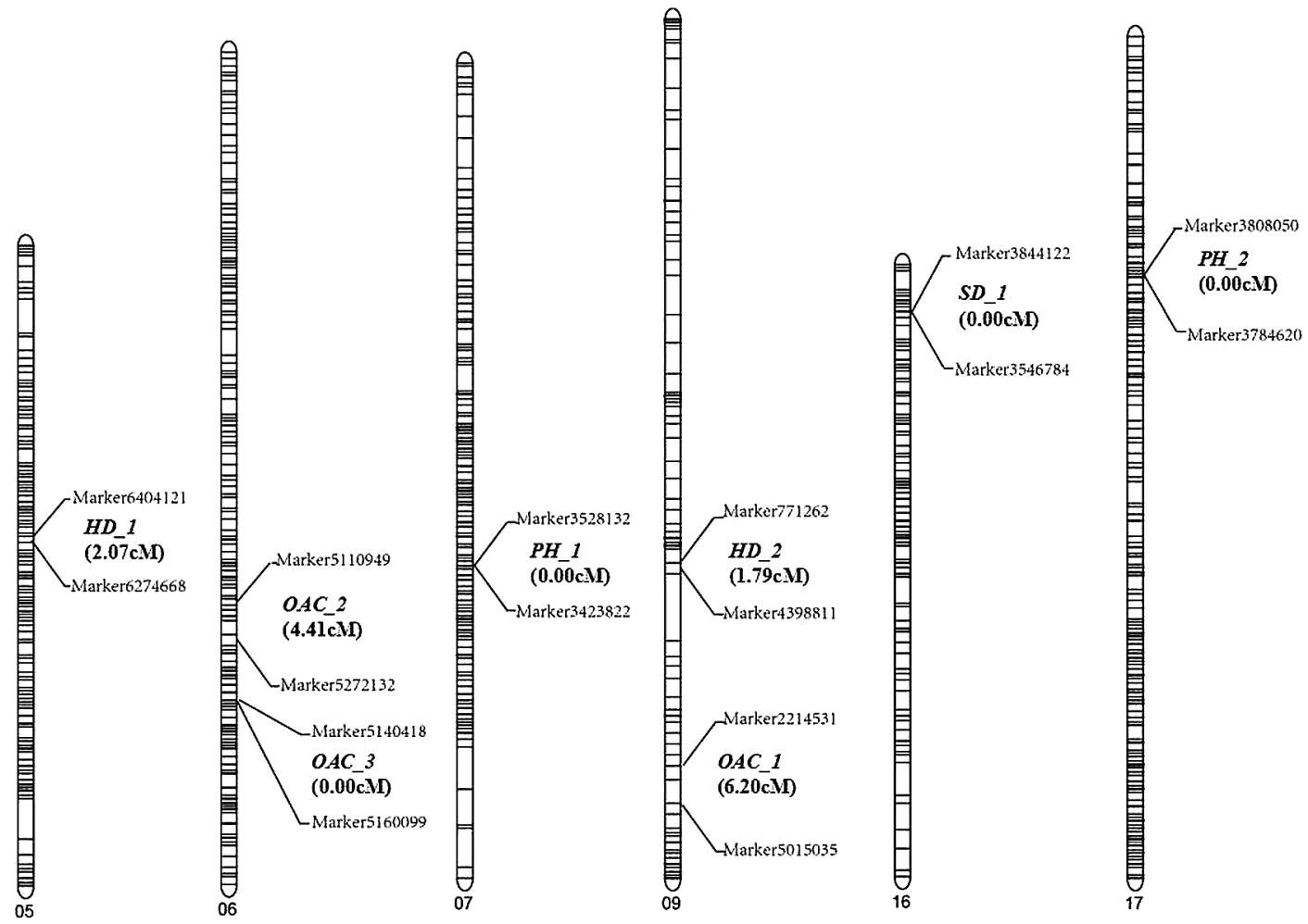

Fig. 5. QTL locations on LGs for PH (plant height), HD (head diameter), SD (stem diameter) and OAC (oleic acid content). The SLAF marker distributions were depicted on the 6 linkage groups based on their genetic positions in centiMorgans (cM). The 8 QTLs along with their interval distances (cM) are shown between the tightly linked SLAF markers on the right side of each linkage group.

\section{Discussion}

Genetic maps play an important role in the identification of functional genes and QTLs of interest. Moreover, these maps are a highly valuable platform for use in comparative analysis of genome structure and for MAS. However, only a few hundred markers are included in most current genetic maps, due to ineffective discovery technologies and high genotyping costs (Liu et al. 2014). The fusion of powerful sequencing technologies with genotyping approaches, including restriction site-associated DNA sequencing (RADseq), genotyping-by-sequencing (GBS) (Davey et al. 2011), and SLAF-seq, allow millions of SNPs to be identified within plant genomes. SLAF-seq has a major advantage in that it allows researchers to first design the experimental system using bioinformatics analysis to screen for fragments of a chosen specific length from a constructed SLAF-seq library. Consequently, SLAF fragment length selection is not plagued by random interruptions and thus achieves better repeatability over RAD-seq and GBS. Moreover, large amounts of sequence information can be generated and whole genome density distributions can be handled using SLAF-seq (Qi et al. 2014) to generate markers with higher density, better consistency, greater effectiveness, and at lower cost than using traditional methods. In this study, $105.60 \mathrm{~Gb}$ of raw data containing $530.50 \mathrm{M}$ reads were generated using SLAF-seq, for a total yield of 343,197 SLAF markers. Of these, 39,589 were polymorphic, with a polymorphism rate of $11.54 \%$. After a series of screenings, 6,136 markers containing 11,980 SNP loci were mapped successfully onto the high-density genetic map. The number of polymorphic markers within each chromosome ranged from 78 to 801, with the final set of SLAF markers obtained covering all sunflower LGs. Furthermore, marker accuracy and integrity determined from haplotype and heat maps were high $(99.79 \%$ ), indicating that marker quality was suitable for construction of a genetic map (Supplemental Data $1,2)$. In conclusion, the number of sunflower polymorphic markers obtained using SLAF-seq technology was higher than obtained using previous methods, further demonstrating that SLAF-seq is an efficient method for obtaining abundant polymorphic markers to rapidly construct high-density genetic maps.

Several genetic maps have been previously reported for sunflower and most were generated using markers obtained using SSR alone or in combination with other markers. Within these maps, the number of mapped markers was relatively small, with relatively large average distances separating adjacent markers. One sunflower genetic map included 459 SSR markers based on $94 \mathrm{~F}_{7}$ recombinant inbred lines (RILs) that mapped to 17 linkage groups. The map length was $1,368.3 \mathrm{cM}$, with a mean density of $3.1 \mathrm{cM} /$ locus (Tang et al. 2002). Meanwhile, another sunflower genetic map incorporating 202 AFLP and 19 SSR markers based on 
$183 \mathrm{~F}_{2}$ individuals was comprised of 18 linkage groups (LGs), with an overall length of $1,751.5 \mathrm{cM}$ and a mean density of $7.4 \mathrm{cM} /$ locus (Kusterer et al. 2004). Another genetic map of sunflower included 202 TRAP and 24 SSR markers based on $120 \mathrm{~F}_{2}$ individuals and was comprised of 17 linkage groups (LGs), with an overall length of $1,597.5 \mathrm{cM}$ and a mean density of one marker per $7.1 \mathrm{cM}$ (Bing et al. 2008). Yet another sunflower genetic map was reported that incorporated 547 markers (231 SSR, 9 ESTSSR, 3 InDels, and 304 AFLPs based on 94 RILs) that mapped to 17 linkage groups (LGs); the map had an overall length of $1,942.3 \mathrm{cM}$ and mean density of one marker per $3.6 \mathrm{cM}$ (Talia et al. 2010). Another sunflower genetic map, containing 88 AFLP and 44 SSR markers derived from 101 $\mathrm{F}_{2}$ individuals, contained 17 LGs and possessed an overall length of $1,490 \mathrm{cM}$ and mean density of one marker per $12.44 \mathrm{cM}$ (Maleki et al. 2014). Recently, the availability of high-throughput genotyping technologies has made it possible to construct even higher density genetic maps. One map incorporated 5,019 SNP markers obtained using RAD tag sequencing of 118 publicly available SSR markers; this map integrated SNP data from three $\mathrm{F}_{2}$ mapping populations and spanned a length of $1,443.84 \mathrm{cM}$, with an average marker spacing of $0.28 \mathrm{cM}$ (Talukder et al. 2014). In the current study, bioinformatics analysis was used in conjunction with the published sunflower genome reference sequence to improve map construction accuracy, resulting in the mapping of 6,136 markers to 17 LGs. The map described here spans a total of $2,221.86 \mathrm{cM}$, with an average inter-marker distance of $0.36 \mathrm{cM}$. Compared to the high-density genetic map previously reported (Talukder et al. 2014), this genetic map contains more markers and spans longer genetic distances.

One challenge to efficient mapping, segregation distortion, is ubiquitous in many species and is observed when the genotypic frequency of a marker deviates from the expected Mendelian ratio. This phenomenon mainly results from gametophyte and/or zygotic selection or chromosomal rearrangements (Menz et al. 2002). Indeed, studies have demonstrated that segregation distortion of a marker can occur in a non-random and consistent distribution pattern, suggesting that the elimination of gametes or zygotes by a lethal factor located within a neighboring region of the marker causes this phenomenon (Mace et al. 2009). In the present study, an $\mathrm{F}_{2}$ mapping population that was employed to construct a linkage map of 6,136 assigned markers only produced 25 markers $(0.41 \%)$ that exhibited significant segregation distortion. The low ratio of segregation distortion observed here may be related to the population type and genetic relationships within the mapping population used in this study. A previous study had indicated that marker distorted regions were present within LGs 4, 9, 11, and 12 in a sunflower map constructed across an $F_{2}$ population (Talukder et al. 2014). In the current study, segregation distortion markers were also distributed on LG4, LG9, and LG11, which indicates that a similar mechanism of skewed segregation may be common to the two studies. Meanwhile, other studies have demonstrated that linkage mapping applications (such as QTL mapping) are not affected by the presence of segregation distortion markers (Xu and Hu 2009, Zhang et al. 2010) and thus may not negatively impact the successful application of high-density genetic map, as developed in this work.

The genetic map described in this study should be useful for three applications. First, the map provides useful data for further QTL mapping. Second, the results of this study should guide molecular marker-assisted breeding of sunflower germplasm and the cloning of related genes. Third, the high-quality genetic map described here will serve as a reference to anchor scaffolds onto chromosomes that will facilitate completion of next-generation sequencing of whole sunflower genomes (Badouin et al. 2017). However, abundant repetitive DNA sequences in sunflower may still hinder assembly accuracy.

Agronomic traits such as plant height, head diameter, and stem diameter are important traits for sunflower breeding. However, genetic studies focused on these traits in sunflower have seldom been reported. Using $\mathrm{F}_{2}$ and $\mathrm{F}_{3}$ populations, 3 significant QTLs for plant height were previously identified that were localized to LG1, LG5, and LG8 (Bert et al. 2003). In addition, 5 QTLs for plant height, 4 QTLs for head diameter, and 5 QTLs for stem diameter have been identified using RILs (Al-Chaarani et al. 2004), while 6 QTLs for plant height were obtained using $\mathrm{F}_{2}$ and $\mathrm{F}_{3}$ populations and were localized to LG8 and LG17 (Yue et al. 2008), 3 QTLs for head diameter were obtained using $F_{2}$ and $\mathrm{F}_{3}$ populations and were localized to LG9, LG13, and LG17 (Yue et al. 2009), and 11 QTLs for plant height and 9 QTLs for head diameter were obtained using RILs (Haddadi et al. 2011).

It has been acknowledged that sunflower oil with high oleic acid content shares positive nutritional qualities with olive oil (Lacombe et al. 2009). Therefore, one important focus of sunflower breeding would be to increase oleic acid content in seed oil. The genetics of oleic acid content in sunflower have been reported by many researchers (AlChaarani et al. 2004, Burke et al. 2005, Ebrahimi et al. 2008, Fernandez-Martinez et al. 1989, Hongtrakul et al. 1998, Lacombe and Berville 2001, Miller et al. 1987, PerezVich et al. 2002). To date, these studies have resulted in the detection of several QTLs influencing oleic acid content (Kusterer et al. 2004, Premnath et al. 2016).

Here we identified 8 QTLs for plant height, head diameter, stem diameter, and oleic acid content. The QTL for stem diameter $\left(S D_{-}\right)$) accounted for more than $15 \%$ of phenotypic variation in this study and will be useful in sunflower plant breeding. Unfortunately, it is difficult to compare QTLs found here with those described in previous reports, due to the unrelated markers used for map construction. However, some QTLs detected in this study, such as $P H \_1$ on LG 7, PH_2 on LG 17, HD_l on LG 5, HD_2 on LG 9, and $O A C \_1$ on LG 9 share LGs with QTLs previously reported. 
Meanwhile, three agronomic traits $(\mathrm{PH}, \mathrm{HD}$, and $\mathrm{SD})$ in the $\mathrm{F}_{2}$ population exhibited normal distribution frequencies in this study, which confirmed that these traits are inherited in a quantitative manner (Fig. 4). However, oleic acid content did not exhibit a normal distribution frequency when we performed QTL mapping for this trait. This result may reflect the fact that a quantitative trait aligns with a normal distribution only under the hypothesis of multiple gene involvement. When the number of QTLs is small and there are a few QTLs with large genetic effects, phenotypic data do not obey a normal distribution, even though the nonnormality of phenotypic data does not affect QTL mapping (Li et al. 2010).

In this study, LOD scores varied from 2.15 to 2.78 among QTLs identified for HP, HD, SD, and OAC and were thus low enough to prompt questioning of the accuracy of QTL identification. In our opinion, there are several reasons for the low LOD scores. First of all, low LOD scores were likely due to the small number of $F_{2}$ individuals used in this study, resulting in reduced detection of small recombination rates and ultimately decreased QTL detection efficiency. In order to verify accuracy of these QTLs and detect the stability of QTLs in this study, next we will performed backcross tests in this population and QTL analysis for plants grown in different environments. Second, in our study we used R/qtl to analyze QTLs, which employs a reference coefficient that differs from that employed by other software (such as MapQTL), contributing to the low LOD scores observed for our QTL analysis results. Third, phenotypic data itself might contain a certain error due to an unknown but important factor that affects LOD scores. Nevertheless, the QTLs identified in this work will be helpful for future mapping of useful sunflower genes.

\section{Acknowledgments}

This research was supported by the Fundamental Research Funds for the Central Universities (2572015EA05), Heilongjiang Academy of Agricultural Sciences innovation fund of China (2014QN020; 2017SJ036), National Characteristic Oil Plants Industry Technology System (CARS-14), National key research project (2017YFD0300505), Northeast Flax Scientific Observation Experimental Station of Ministry of Agriculture and Flax Branch of the National Bast Fiber Germplasm Improvement Center.

\section{Literature Cited}

Al-Chaarani, G.R., L. Gentzbittel, X.Q.Huang and A. Sarrafi (2004) Genotypic variation and identification of QTLs for agronomic traits, using AFLP and SSR markers in RILs of sunflower (Helianthus annuus L.). Theor. Appl. Genet. 109: 1353-1360.

Badouin, H., J.Gouzy, C.J.Grassa, F. Murat, S.E. Staton, L. Cottret, C.Lelandais-Brière, G.L.Owens, S.Carrère, B. Mayjonade et al. (2017) The sunflower genome provides insights into oil metabolism, flowering and asterid evolution. Nature 546: 148-152.

Berry, S.T., A.J.Leon, C.C.Hanfrey, P.Challis, A.Burkholz, S.R.
Barnes, G.K. Rufener, M.Lee and P.D.Caligari (1995) Molecular marker analysis of Helianthus annuus L. 2. Construction of an RFLP linkage map for cultivated sunflower. Theor. Appl. Genet. 91: 195-199.

Bert, P.F., I.Jouan, D. Tourvieille de Labrouhe, F. Serre, J.Philippon, P. Nicolas and F. Vear (2003) Comparative genetic analysis of quantitative traits in sunflower (Helianthus annuus L.). 2. Characterisation of QTL involved in developmental and agronomic traits. Theor. Appl. Genet. 107: 181-189.

Burke, J.M., S.J.Knapp and L.H.Rieseberg (2005) Genetic consequences of selection during the evolution of cultivated sunflower. Genetics 171: 1933-1940.

Davey, J.W., P.A. Hohenlohe, P.D.Etter, J.Q. Boone, J.M. Catchen and M.L.Blaxter (2011) Genome-wide genetic marker discovery and genotyping using next-generation sequencing. Nat. Rev. Genet. 12: 499-510.

Ebrahimi,A., P. Maury, M. Berger, P.S. Kiani, A. Nabipour, F. Shariati, P. Grieu and A. Sarrafi (2008) QTL mapping of seed-quality traits in sunflower recombinant inbred lines under different water regimes. Genome 51: 599-615.

Fernandez-Martinez, J., A. Jimenez, J.Dominguez, J.M. Garcia, R. Garces and M. Mancha (1989) Genetic analysis of the high oleic acid content in cultivated sunflower (Helianthus annuus L.) Euphytica 41: 39-51.

Fuller, G., M.J.Diamond and T.H.Applewhite (1967) High-oleic safflower oil. Stability and chemical modification. J. Am. Oil Chem. Soc. 44: 264-266.

Ganal, M.W., T.Altmann and M.S. Röder (2009) SNP identification in crop plants. Curr. Opin. Plant Biol. 12: 211-217.

Gentzbittel,L., F. Vear, Y.X.Zhang, A. Bervillé and P. Nicolas (1995) Development of a consensus linkage RFLP map of cultivated sunflower (Helianthus annuus L.). Theor. Appl. Genet. 90: 1079-1086.

Gong, D., L.Huang, X.Xu, C.Wang, M.Ren, C.Wang and M.Chen (2016) Construction of a high-density SNP genetic map in fluecured tobacco based on SLAF-seq. Mol. Breed. 36: 100-111.

Haddadi, P., B. Yazdi-samadi， M.R. Naghavi， A. Kalantari， P. Maury and A. Sarrafi (2011) QTL analysis of agronomic traits in recombinant inbred lines of sunflower under partial irrigation. Plant Biotechnol. Rep. 5: 135-146.

Hongtrakul, V., M.B. Slabaugh and S.J.Knapp (1998) A seed specific delta-12 oleate desaturase gene is duplicated, rearranged, and weakly expressed in high oleic acid sunflower lines. Crop Sci. 38: $1245-1249$

Hulke, B.S., C.J. Grassa, J.E. Bowers, J.M. Burke, L. Qi, Z.I. Talukder and L.H. Rieseberg (2015) A unified single nucleotide polymorphism map of sunflower (Helianthus annuus L.) derived from current genomic resources. Crop Sci. 55: 1696-1702.

Jan, C.C., B.A.Vick, J.F.Miller, A.L.Kahler and E.T.Butler (1998) Construction of an RFLP linkage map for cultivated sunflower. Theor. Appl. Genet. 96: 15-22.

Ji, G., Q.Zhang, R. Du, P.Lv, X.Ma, S.Fan, S.Li, S.Hou, Y.Han and G.Liu (2017) Construction of a high-density genetic map using specific-locus amplified fragments in sorghum. BMC Genomics 18: $51-60$

Kosambi,D.D. (1943) The estimation of map distances from recombination values. Ann. Hum. Genet. 12: 172-175.

Kuster,B., B.Rozynek, L.Brahm, M.Prüfe, S.Tzigos, R. Horn and W. Friedt (2004) Construction of a genetic map and localization of major traits in sunflower (Helianthus annuus L.). Helia 27: 15-24.

Lacombe, S. and A. Berville (2001) A dominant mutation for high oleic acid content in sunflower (Helianthus annuus L.) seed oil is 
genetically linked to a single oleate-desaturase RFLP locus. Mol Breed. 8: 129-137.

Lacombe, S., I. Souyris and A.J.Berville (2009) An insertion of oleate desaturase homologous sequence silences via siRNA the functional gene leading to high oleic acid content in sunflower seed oil. Mol. Genet. Genomics 281: 43-54.

Li, B., S. Fan, F.Yu, Y.Chen, S.Zhang, F.Han, S. Yan, L. Wang and J. Sun (2017) High-resolution mapping of QTL for fatty acid composition in soybean using specific-locus amplified fragment sequencing. Theor. Appl. Genet. 130: 1467-1479.

Li, H., L. Zhang and J. Wang (2010) Analysis and answers to frequently asked questions in quantitative trait locus mapping. Acta Agronomica Sinica 36: 918-931.

Liu, D., C. Ma, W.Hong, L.Huang, M.Liu, H.Liu, H.Zeng, D. Deng, H.Xin, J.Song et al. (2014) Construction and analysis of highdensity linkage map using high-throughput sequencing data. PLoS ONE 9: e98855.

Lyu,P., H.F.Yu, Z.X.Yu, Y.H.Zhang, Y.F.Zhang, T.T.Wang and J.H.Hou (2017) Construction of high-density genetic map and QTL mapping for seed germination traits in sunflower under two water conditions. Acta Agronomica Sinica 43: 19-30.

Mace, E.S., J.F. Rami, S.Bouchet, P.E.Klein, R.R.Klein, A.Kilian, P. Wenzl, L.Xia, K. Halloran and D.R. Jordan (2009) A consensus genetic map of sorghum that integrates multiple component maps and high-throughput Diversity Array Technology (DarT) markers. BMC Plant Biol. 9: 13-26.

Maleki,H.H., R. Darvishzadeh and A.Sarrafi (2014) Genetic linkage map and QTL analysis of partial resistance to black stem in sunflower (Helianthus annuus L.). Australas. Plant Path. 43: 205-213.

Menz, M.A., R.R.Klein, J.E.Mullet, J.A.Obert, N.C.Unruh and P.E. Klein (2002) A high-density genetic map of Sorghum bicolor (L.) Moench based on 2926 AFLP, RFLP and SSR markers. Plant Mol. Biol. 48: 483-499.

Miller, J.F., D.C.Zimmerman and B.A. Vick (1987) Genetic control of high oleic acid content in sunflower oil. Crop Sci. 27: 923-926.

Peng, Q., S. Ye, L.Huang, D. Zhang, Y.Liu, J. Wu, Y.Zhang and S. Zhu (2016) Construction of a high-density genetic map in rice by using specific-length amplified fragment sequencing (SLAF-seq) technology. Mol. Plant Breed. 14: 2127-2132.

Perez-Vich,B.，J.M.Fernandez-Martinez， M.Grondona， S.J.Knapp and S.T.Berry (2002) Stearoyl-ACP and oleoyl-PC desaturase genes cosegregate with quantitative trait loci underlying high stearic and high oleic acid mutant phenotypes in sunflower. Theor. Appl. Genet. 104: 338-349.

Premnath,A., M.Narayana, C.Ramakrishnan, S. Kuppusamy and V. Chockalingam (2016) Mapping quantitative trait loci controlling oil content, oleic acid and linoleic acid content in sunflower (Helianthus annuus L.). Mol. Breed. 36: 106.

Qi,Z., L.Huang, R.Zhu, D.Xin, C.Liu, X.Han, H.Jiang, W.Hong, G. Hu, H.Zheng et al. (2014) A high-density genetic map for soybean based on specific length amplified fragment sequencing. PLoS ONE 9: e104871.

Rieseberg, L.H., H.Choi, R. Chan and C. Spore (1993) Genomic map of a diploid hybrid species. Heredity 70: 285-293.

Shang, J., N. Li, N. Li, Y. Xu, S. Ma and J. Wang (2016) Construction of a high-density genetic map for watermelon (Citrullus lanatus L.) based on large-scale SNP discovery by specific length amplified fragment sequencing (SLAF-seq). Sci. Hortic. 203: 38-46.

Sun, X., D.Liu, X.Zhang, W.Li, H.Liu, W.Hong, C.Jiang, N.Guan,
C. Ma, H.Zeng et al. (2013) SLAF-seq: an efficient method of large-scale de novo SNP discovery and genotyping using high-throughput sequencing. PLoS ONE 8: e58700.

Talia, P., V.Nishinakamasu, H.E.Hopp, R.A.Heinz and N.Paniego (2010) Genetic mapping of EST-SSRs, SSR and InDels to improve saturation of genomic regions in a previously developed sunflower map. Electron. J. Biotechnol. doi: 10.2225/vol13-issue6-fulltext-14.

Talukder,Z.I., L. Gong, B.S. Hulke, V.Pegadaraju, Q. Song, Q. Schultz and L. Qi (2014) A high-density SNP map of sunflower derived from RAD-sequencing facilitating fine-mapping of the rust resistance gene $R_{12}$. PLoS ONE 9: e98628.

Tang, S., J.K. Yu, M.B. Slabaugh, D.K. Shintani and S.J. Knapp (2002) Simple sequence repeat map of the sunflower genome. Theor. Appl. Genet. 105: 1124-1136.

Van Ooijen, J.W. (2011) Multipoint maximum likelihood mapping in a full-sib family of an outbreeding species. Genet. Res. (Camb) 93: 343-349.

Van Os, H., P. Stam, R.G.F. Visser and H.J.V.Eck (2005) SMOOTH: a statistical method for successful removal of genotyping errors from high-density genetic linkage data. Theor. Appl. Genet. 112: 187194.

Wang, J., D.J.Lydiate, I.A.Parkin, C.Falentin, R. Delourme, P.W. Carion and G.J.King (2011) Integration of linkage maps for the amphidiploid Brassica napus and comparative mapping with Arabidopsis and Brassica rapa. BMC Genomics 12: 101-120.

Wang, J., K.Su, Y.Guo, H.Xing, Y.Zhao, Z.Liu, K.Li and X.Guo (2017a) Construction of a high-density genetic map for grape using specific length amplified fragment (SLAF) sequencing. PLoS ONE 12: e0181728.

Wang, L., X.Li, L. Wang, H.Xue, J.Wu, H.Yin and S.Zhang (2017b) Construction of a high-density genetic linkage map in pear (Pyrus communis $\times$ Pyrus pyrifolia nakai) using SSRs and SNPs developed by SLAF-seq. Sci. Hortic. 218: 198-204.

$\mathrm{Xu}$, S.Z. and Z.Q.Hu (2009) Mapping quantitative trait loci using distorted markers. Int. J. Plant Genomics 2009: 410825.

Xu, X., R.Xu, B.Zhu, T.Yu, W.Qu, L.Lu, Q.Xu, X. Qi and X.Chen (2015) A high-density genetic map of cucumber derived from specific length amplified fragment sequencing (SLAF-seq). Front. Plant Sci. 5: 768-775.

Yu, J.K., S. Tang, M.B. Slabaugh, A. Heesacker, G.Cole, M.Herring, J. Soper, F. Han, W.C.Chu, D.M. Webb et al. (2003) Towards a saturated molecular genetic linkage map for cultivated sunflower. Crop Sci. 43: 367-387.

Yue, B., B.A. Vick, J.F. Miller, X. Cai and J.Hu (2008) Construction of a linkage map with TRAP markers and identification of QTL for four morphological traits in sunflower (Helianthus annuus L.). Proceedings of 17th International Sunflower Conference, June 8-12, 2008, Cordoba, Spain, p. 655-660.

Yue, B., X.Cai, W. Yuan, B.Vick and J.Hu (2009) Mapping quantitative trait loci (QTL) controlling seed morphology and disk diameter in sunflower (Helianthus annuus L.). Helia 32: 17-36.

Zhang, J., Q. Zhang, T. Cheng, W. Yang, H. Pan, J.Zhong, L. Huang and E. Liu (2015) High-density genetic map construction and identification of a locus controlling weeping trait in an ornamental woody plant (Prunus mume Sieb. et Zucc). DNA Res. 22: 183-191.

Zhang,L., S.Wang, H.Li, Q.Deng, A.Zheng, S.Li, P.Li, Z.Li and J.Wang (2010) Effects of missing marker and segregation distortion on QTL mapping in $F_{2}$ populations. Theor. Appl. Genet. 121: 1071-1082. 